\title{
ANNOTATION
}

\section{Vaccination Against Pertussis}

In the ten years from 194I to $195^{\circ}$ just under 10,000 children died of pertussis in this country. The number of cases which occurred in that period is not accurately known as notification is certainly not complete, but surveys in Britain, Denmark and the United States indicate that between 60 per cent. and 70 per cent. of children suffer from clinically recognizable attacks before they reach school leaving age. In a recent investigation on over 600 cases in non-immunized children between six months and four years of age, it was found that 60 per cent. of the children continued to cough for eight or more weeks, and 36 per cent. continued to cough for more than ten weeks after the onset of symptoms. Some degree of atalectasis is the rule rather than the exception in children with pertussis, and in a few cases the atalectasis is followed by chronic bronchitis or bronchiectasis. In addition to chest complications convulsions are common and encephalopathy, hemiplegia and even progressive amentia have sometimes been recorded.

The newer antibiotics, chloromycetin and aureomycin, have been giving favourable reports in treatment, though it is becoming clear that good results can only be obtained if the drug is administered before the characteristic nocturnal paroxysmal cough has developed.

The causative organism, $H$. pertussis, forms a single antigenic type and it is not surprising that vaccination with killed cultures was attempted shortly after the first isolation of $H$. pertussis by Bordet and Gengou in 1906. On the whole, the reports of the value of vaccination between 1912 and the present time have been favourable, but in a number of carefully controlled studies there was little difference between the incidence or severity of attack in the vaccinated and the unvaccinated groups of children observed.

The first report of the Whooping Cough Committee of the Medical Research Council (Report I95I) explained some of the reasons for the apparent confusion in the voluminous literature on the subject. As a result of the strictly controlled studies carried out in nearly 10,000 children by Medical Officers of Health in association with the Committee it is clear that, given a good vaccine, the incidence of pertussis can be reduced by 80 or 90 per cent. in the vaccinated, and that the severity of the attack in those children who do develop symptoms can also be reduced.
The difficulty at present lies in the definition what is a good vaccine. In the Medical Resear Council's investigations five batches of vaccime from three manufacturers were employed and even with what were thought to be the most effective vaccines obtainable in Britain or Ameried, the best was three or four times as good as the other vaccines tested. Further investigations methods of preparation and of laboratory testing $\rightarrow$ of vaccines are still in progress, and it is hoped that the intracerebral mouse challenge test may de shown to be satisfactory in distinguishing goosd batches from poor. Until such a test has been proved by concurrent laboratory and field studjes it would not be wise to recommend a scheme for whooping cough vaccination similar to that $\overrightarrow{f q r}$ diphtheria immunization. On the other hand, ge evidence of the value of vaccination is such that parents who wish to have their children inoculat should bè able to have it done, and the following temporary recommendations are accepted by practitioners of preventive medicine.

(I) Vaccination should be confined to childrentivefto are fit and well in every way and who are more than four months old. (2) Children with a personal orr family history of central nervous system diseasse should probably not be inoculated. Any child develops convulsions within 72 hours after an jection of vaccine should not be given further inocubations. There have been reports of the very occasional occurrence of convulsions or encephatopathy after vaccination, but the evidence that such cases are due to the vaccine is not conclusive. (3) Plain suspensions of killed bacilli should be usied in preference to alum-precipitated or 'combined diphtheria-pertussis antigens. There is no evideace that alum-precipitation increases the antige potency of pertussis vaccine, and there is at lease a suspicion that combined antigens containing alum are more likely to be followed within four weeks by paralytic poliomyelitis in the inoculated lip $b$ than other antigens. (4) Vaccination should obe postponed in children who are recovering from vitas infections or whose smallpox vaccination scar thas not healed. (5) Three injections (each of 1.0 ccoif plain suspensions are being used) should be given with an interval of one month between injections.

W. C. Cockburn, M.B., Ch.B., D.P界.

Reference

Report (195 I), Brit. Med. F., i, I463. 